\title{
$O$-Acetylation of Peptidoglycan in Neisseria gonorrhoeae. Investigation of Lipid-linked Intermediates and Glycan Chains Newly Incorporated into the Cell Wall
}

\author{
By ANDREW L. LEAR AND HAROLD R. PERKINS* \\ Department of Microbiology, Life Sciences Building, University of Liverpool, \\ Liverpool L69 3BX, UK
}

(Received 1 April 1986; revised 26 May 1986)

\begin{abstract}
Radioactive labelling of the amino sugars in gonococcal peptidoglycan was followed by treatment with Chalaropsis muramidase and TLC separation of the products. Even after very brief periods of labelling $(0.5 \mathrm{~min})$ the peptidoglycan was already cross-linked to some $80 \%$ of the final value and little change occurred within $2 \mathrm{~min}$. The remaining cross-linking was achieved only over a period of about one generation time. Streptomycete endopeptidase was used to show the extent to which new chains were cross-linked to old. Even at the earliest times many cross-linked units contained new material in both moieties and by 3 min there was little distinction in relative labelling, indicating that in Neisseria gonorrhoeae most newly synthesized glycan chains are cross-linked to other new chains rather than to pre-existing peptidoglycan. A model is proposed in which newly polymerized monomer units are predestined either towards dimer formation with other new chains, which are then rapidly $O$-acetylated and not further cross-linked, or towards the formation of trimers and higher oligomers, the latter being a slower process. Although significant $O$-acetylation of peptidoglycan was detectable even at the earliest times, efforts to detect $O$-acetylated lipid intermediates were unsuccessful. The chief lipid intermediate found was apparently the disaccharide-peptide unit linked to undecaprenol.
\end{abstract}

\section{INTRODUCTION}

The peptidoglycan of Neisseria gonorrhoeae has been shown (Hebeler \& Young, 1976) to have a chemical composition of muramic acid, glucosamine, alanine, glutamic acid, diaminopimelic acid in the ratio $1: 1: 2: 1: 1$ and thus to be of type $\mathrm{Al} \gamma$ (Schleifer \& Kandler, 1972). It has been established (Blundell et al., 1980) that unlike in Escherichia coli, approximately $50 \%$ of the muramic acid residues are $O$-acetylated. The immediate response of gonococci to low concentrations of $\beta$-lactam antibiotics is a decrease in $O$-acetylation rather than in peptide crosslinking (Blundell \& Perkins, 1981; Dougherty, 1985). Using short pulses of radioactive glucosamine added to the growth medium, we have been able to follow the progress of incorporation of precursor into $O$-acetylated and non- $O$-acetylated subunits in SDS-insoluble peptidoglycan (Lear \& Perkins, 1983). In these experiments peptidoglycan was isolated from culture samples taken at intervals after addition of radioactivity and was then digested with Chalaropsis muramidase B (Hash \& Rothlauf, 1967). The resultant fragments were separated by TLC (Martin \& Gmeiner, 1979) into monomers (disaccharide peptide), dimers (bisdisaccharide cross-linked peptide) and oligomers [unresolved in this system, but consisting mainly of trimers (Rosenthal et al., 1980)]. With labelling periods from $3 \mathrm{~min}$ to $90 \mathrm{~min}$ it was shown that newly synthesized peptidoglycan was both less $O$-acetylated and less cross-linked than mature peptidoglycan.

Cross-linking very rapidly ( $3 \mathrm{~min}$ ) achieved $80 \%$ of its final value, but the remainder of the process was much slower, taking a further $60 \mathrm{~min}$. $O$-Acetylation rose quickly over $10 \mathrm{~min}$ to about $65 \%$ of its maximum, the process being completed by $60 \mathrm{~min}$. We concluded that newly 
synthesized peptidoglycan underwent a maturation process involving both further peptide cross-linking and $O$-acetylation. Peptidoglycan maturation involving increases in cross-linking has been observed in E. coli (de Pedro \& Schwarz, 1981) whilst increases in both cross-linking and $O$-acetylation have been reported in Proteus mirabilis (Gmeiner \& Kroll, 1981) and $N$. gonorrhoeae (Lear \& Perkins, 1983; Dougherty, 1983a). However, no study to date has resolved the question of whether all or only a proportion of the $O$-acetyl groups are added to newly synthesized peptidoglycan after its insertion into the cell wall. We have addressed this problem in two ways: first, by investigating the peptidoglycan labelled with very short pulses $(0.5-2 \mathrm{~min})$ of radioactive glucosamine, and second, by isolating the lipid $\left(\mathrm{C}_{55}\right.$-undecaprenol) -linked precursor of peptidoglycan (Higashi et al., 1967, 1970). Very early $O$-acetylation could conceivably take place at the lipid-intermediate stage of peptidoglycan biosynthesis, as for instance has been shown for amidation (Siewert \& Strominger, 1968).

\section{METHODS}

Organisms and growth conditions. Neisseria gonorrhoeae strain 1L260 (Brown \& Perkins, 1979), grown overnight on chocolate agar plates, was used to inoculate proteose peptone no. 3 (Difco) liquid medium, with pyruvate as carbon source (Blundell \& Perkins, 1981). Cultures were incubated with shaking at $37^{\circ} \mathrm{C}$.

Radioactive labelling: isolation and analysis of peptidoglycan. Cultures $(75 \mathrm{ml})$ were grown to $\mathrm{OD}_{675} 0.2$. A $10 \mathrm{ml}$ sample was transferred to a separate flask for radioactive labelling, and incubation of this and the parent culture continued. Then either $25 \mu \mathrm{Ci}(0.925 \mathrm{MBq})$ of $\mathrm{D}-\left[6-{ }^{3} \mathrm{H}\right] \mathrm{glucosamine}$ hydrochloride $\left[40 \mathrm{Ci} \mathrm{mmol}^{-1}\right.$, $1.48 \mathrm{TBq} \mathrm{mmol}{ }^{-1}$, Amersham] $\mathrm{ml}^{-1}$ (for short-term experiments, series 1) or $1.25 \mu \mathrm{Ci}(46 \mathrm{kBq})$ of $\mathrm{D}-[1-$ ${ }^{14} \mathrm{Clglucosamine}$ hydrochloride [ $54 \mathrm{mCi}(2 \mathrm{GBq}) \mathrm{mmol}^{-1}$, Amersham] $\mathrm{ml}^{-1}$ (for longer-term experiments, series 2) was added to the smaller culture at $\mathrm{OD}_{675} 0 \cdot 3$, and the culture was swirled rapidly to ensure thorough mixing. Samples $\left(2 \mathrm{ml}\right.$ ) were added to an equal volume of $10 \%(\mathrm{w} / \mathrm{v}) \mathrm{SDS}$ and heated at $100^{\circ} \mathrm{C}$ for $20 \mathrm{~min}$ (Blundell et al., $1980)$. Samples $(10 \mathrm{ml})$ of the parent culture were similarly treated. Incorporation of radioactivity was measured by filtering $100 \mu$ l volumes of labelled samples on glass microfibre discs (GF/C, Whatman), which were then washed and used for measurement of radioactivity (Blundell \& Perkins, 1981). SDS-insoluble peptidoglycan was isolated from the labelled culture samples after each had been mixed with unlabelled material from samples of the parent culture. Insoluble material collected by centrifugation (Blundell \& Perkins, 1981) was digested with Chalaropsis muramidase $B$ and the fragments were separated by TLC in isobutyric acid/1 M-ammonia (5:3, v/v) (Martin \& Gmeiner, 1979). After drying, TLC plates were sprayed with En $^{3}$ Hance (New England Nuclear) and spots were detected by fluorography. Spots identified by comparison with previously identified markers were cut out and their radioactivity was measured in a scintillation counter.

Endopeptidase treatment of mono-O-acetylated dimers. After scintillation counting, strips of TLC corresponding to spots identified as mono- $O$-acetylated dimer were washed three times with toluene to remove scintillant and then were eluted with water. After drying over $\mathrm{NaOH}$ under reduced pressure, samples were resuspended in $75 \mu \mathrm{l}$ $0.5 \mathrm{M}-\mathrm{Tris} / \mathrm{HCl} \mathrm{pH} 7.5$ containing $5 \mathrm{mM}-\mathrm{MgCl}_{2}$ and $15 \mu \mathrm{g}$ DD-carboxypeptidase from Streptomyces albus $\mathrm{G}$ (Ghuysen et al., 1970), and incubated at $37^{\circ} \mathrm{C}$ overnight. This enzyme has been shown to have endopeptidase activity (Leyh-Bouille et al., 1970) and will cleave both $O$-acetylated and non-O-acetylated dimers derived from $N$. gonorrhoeae peptidoglycan into their constituent subunits (unpublished results). The products of endopeptidase treatment were separated by TLC, detected and quantified as above.

Isolation of the $C_{55}$-undecaprenol-linked precursors of peptidoglycan. Cultures (21) were grown to $\mathrm{OD}_{675} 0 \cdot 4$. D-[1$\left.{ }^{14} \mathrm{C}\right]$ Glucosamine hydrochloride $\left[56.8 \mu \mathrm{Ci} \mathrm{mmol}^{-1}, 2 \cdot 10 \mathrm{GBq} \mathrm{mmol}^{-1}\right.$; Amersham] was added (final concentration $0.08 \mu \mathrm{Ci} \mathrm{ml}^{-1}$ ) and incubation continued for $10 \mathrm{~min}$. Cultures were then cooled rapidly by pouring onto 1.21 of crushed frozen liquid medium, and cells were harvested by centrifugation at $5200 \mathrm{~g}$ for $20 \mathrm{~min}$ at $2{ }^{\circ} \mathrm{C}$. The pellets were quickly resuspended in $190 \mathrm{ml}$ boiling water and heated at $100^{\circ} \mathrm{C}$ for $10 \mathrm{~min}$. After cooling, cellular material was collected by centrifugation $(115000 \mathrm{~g}, 90 \mathrm{~min})$, resuspended in $50 \mathrm{ml} 50 \mathrm{~mm}$-sodium phosphate buffer $\mathrm{pH} 7 \cdot 2$ and extracted with an equal volume of butan-1-ol/6 M-pyridinium acetate $\mathrm{pH} 4 \cdot 2(2: 1, \mathrm{v} / \mathrm{v})$ to remove lipid intermediate (Anderson et al., 1967). The aqueous phase was re-extracted twice with butan-1-ol. The combined butan-1-ol extracts were washed three times with water and dried by evaporation under reduced pressure. The residue was resuspended in chloroform/methanol $(1: 1, \mathrm{v} / \mathrm{v})$, applied to the origin of a paper chromatogram (3 MM, Whatman) and fractionated by descending chromatography in isobutyric acid/1 Mammonia $(5: 3, \mathrm{v} / \mathrm{v})$ for $18 \mathrm{~h}$ (Anderson et al., 1967). Sample tracks were cut into $0.5 \mathrm{~cm}$ strips and radioactivity was detected by scintillation counting. Of the two major peaks, peak $I$ ran just behind the solvent front $\left(R_{F} 0.9\right)$ as expected for lipid-linked intermediates (Anderson et al., 1967). Peak II $\left(R_{F} 0 \cdot 7\right)$ was unidentified, but on hydrolysis $\left(4 \mathrm{M}-\mathrm{HCl}, 4 \mathrm{~h}, 100^{\circ} \mathrm{C}\right.$ ) was found to contain no muramic acid (results not shown) and was therefore deemed to be neither peptidoglycan nor one of its precursors. Chromatogram strips corresponding to peak I were washed with toluene to remove scintillant, then immersed in $0.1 \mathrm{M}-\mathrm{HCl}$ and heated to $100^{\circ} \mathrm{C}$ for $10 \mathrm{~min}$ to hydrolyse the 
pyrophosphoryl link between the lipid and glycan portions of lipid intermediate (Siewert \& Strominger, 1968). Solubilized material was eluted with water and fractionated by descending paper chromatography ( $3 \mathrm{MM}$, Whatman) in butan-1-ol/acetic acid/water (4:1:5, by vol., upper phase) for $72 \mathrm{~h}$. Radioactive spots were detected as described before, eluted with water, hydrolysed with $4 \mathrm{M}-\mathrm{HCl}$ at $100^{\circ} \mathrm{C}$ for $4 \mathrm{~h}$ in sealed tubes, and the liberated sugars separated by descending paper chromatography ( $3 \mathrm{MM}$, Whatman) in butan-1-ol/acetic acid/water for $34 \mathrm{~h}$.

Source of marker compounds. Monomer and $O$-acetylated monomer were obtained essentially as by Chapman \& Perkins (1983). Other gonococcal peptidoglycan fragments were purified as described by Blundell \& Perkins (1981). $N$-Acetylmuramyl pentapeptide, a gift of N. McKendrick, was made from Bacillus licheniformis UDP- $N$ acetylmuramyl pentapeptide (Ward, 1974) by hydrolysis with $0.1 \mathrm{M}-\mathrm{HCl}$ at $100{ }^{\circ} \mathrm{C}$ for $10 \mathrm{~min}$. Muramic acid was purchased from Sigma. Unlabelled marker compounds were detected on chromatograms by spraying with $0.4 \%$ (w/v) ninhydrin (Sigma) in acetone, or silver nitrate/sodium hydroxide (Trevelyan et al., 1950).

Enzymes. Chalaropsis muramidase B was given by Dr J. B. Ward and DD-carboxypeptidase from $S$. albus $\mathrm{G}$ by Professor J.-M. Ghuysen.

\section{RESULTS}

Cultures incubated with radioactive glucosamine incorporated label very rapidly into SDSinsoluble material. After as little as $0.5 \mathrm{~min}$ significant amounts of radioactivity appeared in the cell wall. The composition of newly synthesized peptidoglycan as deduced from the proportions of radioactivity found in the various Chalaropsis muramidase fragments separated by TLC is shown in Table 1. The degrees of $O$-acetylation and peptide cross-linking were calculated as before (Blundell \& Perkins, 1981). The TLC method does not distinguish between $O$-acetylated monomers and monomers in which the anhydro form of muramic acid is present (Swim et al., 1983 ) but there is no reason to suppose that this would involve more than a small proportion of the units at any time point (Blundell \& Perkins, 1985 ). Calculations of cross-linking would in any case be unaffected. Because of the possibility of asymmetric labelling of the mono- $O$ acetyldimer fraction in newly synthesized peptidoglycan (Gmeiner \& Kroll, 1981), mono-Oacetyldimer spots were eluted from TLC plates and further digested by $S$. albus G DDcarboxypeptidase. Mono- $O$-acetylated dimer, isolated from cultures of $N$. gonorrhoeae labelled with radioactive glucosamine for about two generation times, when treated with this enzyme, yields monomer and $O$-acetylated monomer with approximately equal amounts of radioactivity (the minor difference was assumed to be due to chemical loss of $O$-acetyl during the digestion: cf. Table 1). In cultures pulsed for $0.5-1.5 \mathrm{~min}$ this ratio was about $2: 1$, decreasing to $5: 3$ for pulses of $2 \mathrm{~min}$ and approaching $1: 1$ within $15 \mathrm{~min}$ (Table 1). The proportion of newly synthesized subunits in the dimer fraction that were $O$-acetylated was also calculated from these results (Table 1).

After 0.5 min monomer $\mathrm{O}$-acetylation was only $22 \%$ of its final, mature value whilst that of newly synthesized dimers was $42 \%$ of maximum. The latter proportion also increased somewhat within the next 2-3 min. The degree of peptide cross-linking was calculated after allowance had been made for the lower specific activity of the $O$-acetylmonomer moiety of the mono- $O$ acetyldimer observed at short labelling times (see Table 1). At $0.5 \mathrm{~min}$ the degree of crosslinking was $28 \cdot 6,80 \%$ of the final value of 35.6 observed after long-term incorporation $(90 \mathrm{~min})$.

The presence of $O$-acetyl groups in newly synthesized SDS-insoluble peptidoglycan within $0.5 \mathrm{~min}$ of the bacteria being pulsed with glucosamine prompted us to look for $O$-acetylation of the lipid-linked intermediates of peptidoglycan synthesis. Lipid-linked precursor was isolated as fast-migrating glucosamine-labelled material $\left(R_{F} 0.9\right)$ on paper chromatography of a butan-1ol/pyridinium acetate extract of $N$. gonorrhoeae. To investigate the glycan portion of the molecule, the pyrophosphoryl link between lipid and glycan was broken by brief hydrolysis with $0 \cdot 1 \mathrm{M}-\mathrm{HCl}$. Parallel experiments showed that these conditions produced no significant hydrolytic removal of $O$-acetyl groups from peptidoglycan fragments (data not shown) and Ward \& Perkins (1973) have shown that they do not result in hydrolysis of glycan chains. The fragments released were characterized by further paper chromatography in butan-1-ol/acetic acid/water (Fig. 1). Over $80 \%$ of the radioactivity was recovered in a single peak with a chromatographic mobility similar to that of disaccharide peptides (non- $O$-acetylated 


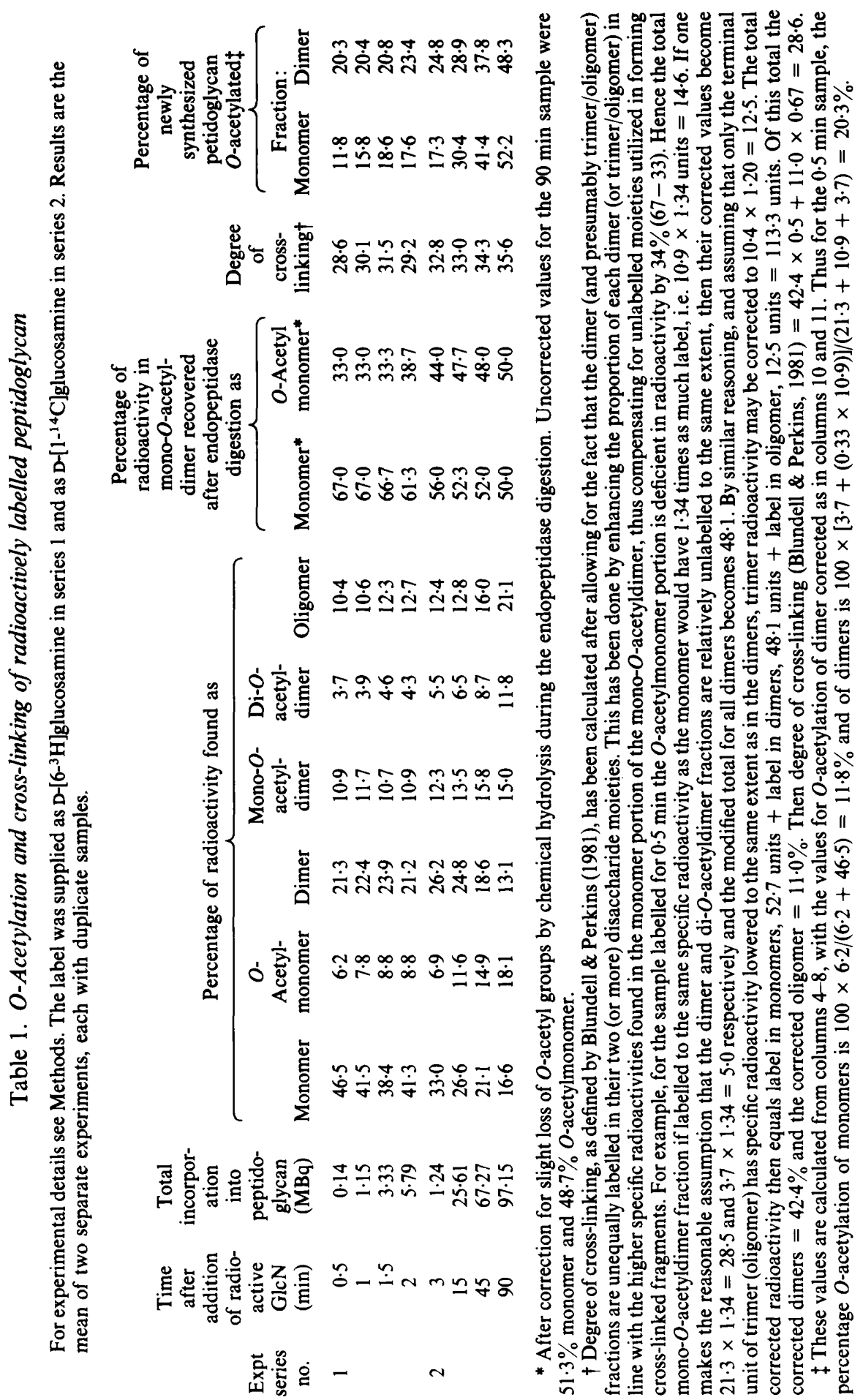




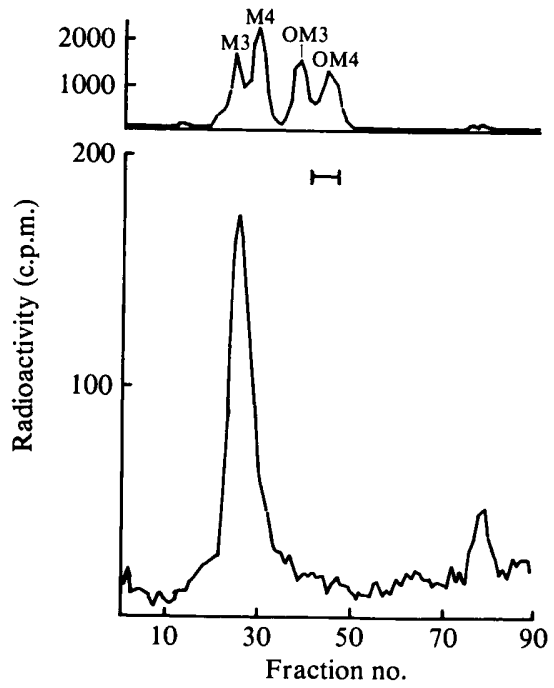

Fig. 1. Descending paper chromatography of radioactivity solubilized by acid hydrolysis $(0 \cdot 1 \mathrm{M}-\mathrm{HCl}$, $10 \mathrm{~min}, 100^{\circ} \mathrm{C}$ ) from lipid intermediate labelled in the glycan portion of the molecule by $\mathrm{D}-[1-$ ${ }^{14} \mathrm{Clglucosamine}$. Solvent : butan-1-ol/acetic acid/water ( $4: 1: 5$, by vol., upper phase). Chromatogram tracks were cut into $0.5 \mathrm{~cm}$ strips, for measurement of radioactivity in a scintillation counter. Sample (lower trace) and standard monomers (upper trace) were compared. Unlabelled $N$-acetylmuramylpentapeptide $(\longmapsto)$ was detected by spraying with ninhydrin. In this system monomers (disaccharide peptides) from $N$. gonorrhoeae ran as four spots, in order from the origin : disaccharide tripeptide (M3) disaccharide tetrapeptide (M4), followed by their respective $O$-acetylated derivatives (OM3, OM4).

monomers) but considerably slower than that of $O$-acetylated disaccharide peptides $(O$ acetylated monomers). A minor faster-running peak had a relative chromatographic mobility $\left(R_{\mathrm{C} 6}\right)$ of $2 \cdot 67$, similar to that of $N$-acetylglucosamine $\left(R_{\mathrm{C} 6} 2 \cdot 6\right)$ (Chapman \& Perkins, 1983). No peak was detected with a chromatographic mobility corresponding to that of $O$-acetylated monomers.

Hydrolysis of the major peak with $4 \mathrm{M}-\mathrm{HCl}$ yielded two radioactive spots on descending paper chromatography in butan-1-ol/acetic acid/water, corresponding to glucosamine and muramic acid. The muramic acid spot contained only $37 \%$ of the activity found in the glucosamine spot. However, no carrier was added and it is known that very small amounts of muramic acid are relatively labile under the conditions used for hydrolysis and appreciable loss would be expected. Rosenthal (1979) observed similar losses in some experiments. Parallel hydrolysis of the fasterrunning peak $\left(R_{\mathrm{C} 6} 2.67\right)$ showed that it contained no labelled material with the mobility of muramic acid.

\section{DISCUSSION}

Previous work showing that peptidoglycan newly synthesized by $N$. gonorrhoeae is less crosslinked and less $O$-acetylated than mature peptidoglycan (Lear \& Perkins, 1983) has been extended to very short labelling times. Pulses of radioactive glucosamine as short as $0.5 \mathrm{~min}$ labelled SDS-insoluble peptidoglycan, which was shown to be both cross-linked and $O$ acetylated to a significant degree. Transpeptidation was especially rapid, with the degree of cross-linking being $80 \%$ of that of mature peptidoglycan after a $0.5 \mathrm{~min}$ pulse. There was little increase in cross-linking over the 2 min time course, suggesting that the slower secondary transpeptidation noted previously in periods greater than $3 \mathrm{~min}$ (Lear \& Perkins, 1983) did not commence appreciably before that time. Whether there was a delay in the onset of secondary cross-linking extending as long as $10 \mathrm{~min}$, as observed in E. coli by de Pedro \& Schwarz (1981), cannot be deduced from our experiments. 
During periods up to $2 \mathrm{~min}, O$-acetylation was much less near to completion than crosslinking, but did not approach zero even at the shortest labelling time. $O$-Acetylation of newly synthesized cross-linked peptidoglycan was considerably greater than that of the uncross-linked material (Table 1). These differences diminished with time but were still perceptible even in the 3-10 min period (Lear \& Perkins, 1983). The fact that, throughout the early stage of synthesis, the cross-linked peptidoglycan was consistently more highly $O$-acetylated than the uncrosslinked polymer of necessity implied that pre-formed dimers were preferentially $O$-acetylated. However, the pattern of $O$-acetylation does not suggest that all $O$-acetylated monomers arise by cleavage of $O$-acetylated dimers, even though a suitable endopeptidase is known to exist (Chapman \& Perkins, 1983; Blundell \& Perkins, 1985a). The only way for such a mechanism to operate would be if primary synthesis, cross-linking, $O$-acetylation and endopeptidase action all took place within about $1 \mathrm{~min}$, whereafter the endopeptidase ceased for some reason to act, since otherwise one would expect more evidence of continuing peptide splitting, which did not appear to occur. Our experiments, in which pre-formed mono- $O$-acetylated dimer was split into its constituent monomer and $O$-acetylated monomer units by the use of exogenous endopeptidase, clearly showed that some of the latter subunits were derived from unlabelled and therefore biosynthetically 'old' glycan chains. Unlike in $P$. mirabilis, where most of the mono-Oacetylated dimer fraction carried radioactivity exclusively in the non- $O$-acetylated subunit (Gmeiner \& Kroll, 1981), we found that only a relatively small proportion of the $O$-acetylated subunits were derived from 'old' chains. Asymmetric labelling of the mono- $O$-acetylated dimer fraction was greatly diminished after $3 \mathrm{~min}$ and had virtually disappeared by $15 \mathrm{~min}$ (Table 1). We therefore conclude that in $N$. gonorrhoeae a large proportion of the newly synthesized glycan chains are cross-linked to other newly synthesized chains rather than to pre-existing older unlabelled chains with their higher degree of $O$-acetylation. This would mean that, once begun, a glycan chain would become inaccessible to further dimer formation soon after $3 \mathrm{~min}$, a conclusion in accord with the very high proportion (92\%) of the final degree of cross-linking achieved within the same period.

The secondary cross-linking during the maturation period was evidently involved mainly with the formation of oligomers, while the proportion of dimers remained unchanged (Table 1 and Lear \& Perkins, 1983). A possible explanation for this would be that newly polymerized monomer units are predestined, either by local factors in the gonococcal wall or by their topography within the growing cell, e.g. septal rather than peripheral, to follow one of two pathways. The first, and major, route would involve rapid dimer formation mainly by crosslinkage to similar chains being simultaneously synthesized. These dimer units would undergo relatively rapid $O$-acetylation but would not become further cross-linked to form trimers or higher oligomers. The second, minor route would be the main channel by which the trimer/oligomer component was formed. Here monomer units would be programmed ab initio to form multiple cross-links and would not draw on the corpus of dimer units to make trimers/oligomers. This second route to cross-linking would be relatively slow and would account for the fact that pulse-labelled dimer units do not contribute to trimer/oligomer formation during a chase period, the radioactive label arising in the latter components solely at the expense of monomers (Lear \& Perkins, 1983).

The presence of a significant degree of $O$-acetylation of SDS-insoluble peptidoglycan even after the shortest labelling periods led us to investigate the possibility that $O$-acetylation occurred at the lipid-intermediate stage. Extraction of the lipid intermediates by the procedure of Anderson et al. (1967) yielded material with the characteristics of undecaprenol-linked peptidoglycan precursor. This major lipid intermediate of peptidoglycan synthesis contained the disaccharide-peptide (presumably pentapeptide) but there was no evidence for a corresponding $O$-acetylated intermediate. The absence of any detectable $O$-acetylation of the lipid-linked intermediates would suggest that the initial $O$-acetylation occurs in conjunction with the primary transpeptidation whereby newly synthesized peptidoglycan is cross-linked into the cell wall. The rapidity of these processes implies that they may all occur at the surface of a single enzyme or enzyme-complex. The suggestion that in $E$. coli two of these activities (transglycosylation and transpeptidation) may be dual functions of single enzymes (penicillin- 
binding proteins PBP1a, 1b or 3) is already well known (Ishino et al., 1980, 1981; Suzuki et al., $1980)$. So far as $N$. gonorrhoeae is concerned, Dougherty $(1983 b, 1985)$ has suggested a close correlation between the function of PBP2 and the process of $O$-acetylation. A pen $A$ strain, in which PBP2 had much less affinity for penicillin than the wild-type, also showed less decrease in $O$-acetylation in response to low concentrations of penicillin (Dougherty, 1985). Like ourselves, he suggests a close association between $O$-acetylation and peptidoglycan synthesis as a possible explanation for his results. However, in our hands, a penA2 strain (FA102) behaved similarly to the wild-type (FA19) in showing a $40 \%$ decrease in $O$-acetylation of the monomer fraction in response to concentrations of benzylpenicillin approximately equal to the respective MIC values (J. K. Blundell \& H. R. Perkins, unpublished results). It may be, therefore, that penA resistant strains differ in their response, either via PBP2 or by some other mechanism.

Our experiments on the relative effects of penicillin on early and late $O$-acetylation and crosslinking will form the substance of a later communication.

\section{REFERENCES}

ANDerson, J. S., Matsuhash, M., Haskin, M. A. \& Strominger, J. L. (1967). Biosynthesis of the peptidoglycan of bacterial cell wall. II. Phospholipid carriers in the reaction sequence. Journal of Biological Chemistry 242, 3180-3190.

BLUNDELL, J. K. \& PERKINS, H. R. (1981). Effects of $\beta$ lactam antibiotics on peptidoglycan synthesis in growing Neisseria gonorrhoeae, including changes in the degree of $O$-acetylation. Journal of Bacteriology 147, 633-641.

Blundell, J. K. \& Perkins, H. R. (1985a). Selectivity for $O$-acetylated peptidoglycan during endopeptidase action by permeabilized Neisseria gonorrhoeae. FEMS Microbiology Letters 30, 67-69.

Blundell, J. K. \& Perkins, H. R. (1985b). The peptidoglycan of Neisseria gonorrhoeae, with or without $\boldsymbol{O}$-acetyl groups, contains anhydromuramyl residues. Journal of General Microbiology 131, 33973400.

Blundell, J. K., Smith, G. J. \& Perkins, H. R. (1980). The peptidoglycan of Neisseria gonorrhoeae: $O$ acetylation and lysozyme sensitivity. FEMS Microbiology Letters 9, 259-261.

Brown, C. A. \& Perkins, H. R. (1979). In vitro synthesis of peptidoglycan by $\beta$-lactam-sensitive and -resistant strains of Neisseria gonorrhoeae: effects of $\beta$-lactam and other antibiotics. Antimicrobial Agents and Chemotherapy 16, 28-36.

Chapman, S. J. \& Perkins, H. R. (1983). Peptidoglycan-degrading enzymes in ether-treated cells of Neisseria gonorrhoeae. Journal of General Microbiology 129, 877-883.

DOUGHERTY, T. J. (1983a). Synthesis and modification of the peptidoglycan in Neisseria gonorrhoeae. FEMS Microbiology Letters 17, 51-53.

DOUgherty, T. J. (1983b). Peptidoglycan biosynthesis in Neisseria gonorrhoeae strains sensitive and intrinsically resistant to $\beta$-lactam antibiotics. Journal of Bacteriology 153, 429-435.

DOUGHERTY, T. J. (1985). Involvement of a change of penicillin target and peptidoglycan structure in lowlevel resistance to $\beta$-lactam antibiotics in Neisseria gonorrhoeae. Antimicrobial Agents and Chemotherapy 28, 90-95.

GhuYsen, J.-M., Leyh-Bouille, M., Bonaly, R., Nieto, M., Perkins, H. R., Schleifer, K. H. \& KANDLER, O. (1970). Isolation of DD-carboxypepti- dase from Streptomyces albus G culture filtrates. Biochemistry 9, 2955-2961.

GMeINER, J. \& KROLL, H.-P. (1981). Murein biosynthesis and $O$-acetylation of $N$-acetylmuramic acid during the cell division cycle of Proteus mirabilis. European Journal of Biochemistry 117, 171-177.

Hash, J. H. \& Rothlauf, M. V. (1967). The N,Odiacetyl-muramidase of Chalaropsis species. Purifcation and crystallization. Journal of Biological Chemistry 242, 5586-5590.

Hebeler, H. H. \& Young, F. E. (1976). Chemical composition and turnover of peptidoglycan in Neisseria gonorrhoeae. Journal of Bacteriology 126, 1180-1185.

Higashi, Y., Strominger, J. L. \& Sweeley, C. C. (1967). Structure of a lipid intermediate in cell wall peptidoglycan synthesis: a derivative of a $\mathrm{C}_{55}{ }^{-}$ isoprenoid alcohol. Proceedings of the National Academy of Sciences of the United States of America 57, 1878-1884.

Higashi, Y., Strominger, J. L. \& Sweeley, C. C. (1970). Biosynthesis of the peptidoglycan of bacterial cell walls. XXI: Isolation of free $\mathrm{C}_{55}$-isoprenoid alcohol and of lipid intermediates in peptidoglycan synthesis from Staphylococcus aureus. Journal of Biological Chemistry 245, 3697-3702.

Ishino, F. \& Matsuhashi, M. (1981). Peptidoglycan synthetic enzyme activities of highly purified penicillin-binding protein 3 in Escherichia coli: a septumforming reaction sequence. Biochemical and Biophysical Research Communications 101, 905-911.

Ishino, F., Mitsui, K., TAMaKI, S. \& Matsuhashi, M. (1980). Dual enzyme activities of cell wall peptidoglycan synthesis, peptidoglycan transglycosylase and penicillin-sensitive transpeptidase in purified preparations of Escherichia coli penicillin-binding protein 1A. Biochemical and Biophysical Research Communications 97, 287-293.

Lear, A. L. \& Perkins, H. R. (1983). Degrees of $O$ acetylation and cross-linking of the peptidoglycan of Neisseria gonorrhoeae during growth. Journal of General Microbiology 129, 885-888.

LeYh-Bouille, M., GhuYSEN, J.-M., Bonaly, R., Nieto, M., Perkins, H. R., Schleiper, K. H. \& KANDLER, O. (1970). Substrate requirements of the Streptomyces albus G DD-carboxypeptidase. Biochemistry 9, 2961-2970. 
Martin, H. H. \& Gmeiner, J. (1979). Modification of peptidoglycan structure by penicillin action in cell walls of Proteus mirabilis. European Journal of Biochemistry 95, 487-495.

De Pedro, M. A. \& Schwarz, U. (1981). Heterogeneity of newly inserted and pre-existing murein in the sacculus of Escherichia coli. Proceedings of the National Academy of Sciences of the United States of America 78, 5856-5866.

RosentHAL, R. S. (1979). Release of soluble peptidoglycan from growing gonococci: hexaminidase and amidase activities. Infection and Immunity 24, 869878.

Rosenthal, R. S., Wright, R. M. \& Sinha, R. K. (1980). Extent of peptide cross-linking in the peptidoglycan of Neisseria gonorrhoeae. Infection and Immunity 28, 867-875.

Schleifer, K. H. \& Kandler, O. (1972). Peptidoglycan types of bacterial cell walls and their taxonomic implications. Bacteriological Reviews 36, 407-477.

Siewert, G. \& STRominger, J. L. (1968). Biosynthesis of the peptidoglycan of bacterial cell walls. Formation of the isoglutamine amine group in the cell walls of Staphylococcus aureus. Journal of Biological Chemistry 243, 783-790.
Suzuki, H., van Heijenoort, Y., Tamura, T., Mizoguchi, J., Hirota, Y. \& van Heijenoort, J. (1980). In vitro peptidoglycan polymerization catalysed by penicillin binding protein $1 \mathrm{~B}$ of Escherichia coli K12. FEBS Letters 110, 245-249.

Swim, S. C., GFell, A., WIlde, C. E., III \& Rosenthal, R. S. (1983). Strain distribution in extents of lysozyme resistance and $O$-acetylation of gonococcal peptidoglycan determined by high performance liquid chromatography. Infection and Immunity 42, 446-452.

Trevelyan, W. E., Proctor, D. P. \& Harrison, J. S. (1950). Detection of sugars on paper chromatograms. Nature, London 166, 444-445.

WARD, J. B. (1974). The synthesis of peptidoglycan in an autolysin deficient mutant of Bacillus licheniformis NCTC 6346 and the effects of $\beta$-lactam antibiotics, bacitracin and vancomycin. Biochemical Journal 141, 227-241.

WARD, J. B. \& PERKINS, H. R. (1973). The direction of glycan synthesis in a bacterial peptidoglycan. Biochemical Journal 135, 721-728. 